\title{
APTITUD COMBINATORIA GENERAL Y ESPECÍFICA DE CARACTERES AGRONÓMICOS EN LÍNEAS DE MAÍZ (Zea mays L.) DE BAJA ENDOGAMIA
}

\section{GENERAL AND SPECIFIC COMBINING ABILITY FOR AGRONOMIC TRAITS IN PARTIALLY INBRED LINES OF MAIZE (Zea mays L.)}

Francisco Cervantes-Ortiz ${ }^{1 *}$, Janianny Hernández-Esparza ${ }^{1}$, J. Guadalupe García-Rodríguez ${ }^{1}$, J. Antonio Rangel-Lucio ${ }^{2}$, Enrique Andrio-Enríquez ${ }^{1}$, Mariano Mendoza-Elos ${ }^{1}$, Gilberto RodríguezPérez $^{1}$ y Daniel Rodríguez-Mercado ${ }^{1}$

${ }^{1}$ Tecnológico Nacional de México, Instituto Tecnológico de Roque, Km. 8 Carretera Celaya-J. Rosas, sin número, Celaya, Guanajuato, México. C.P. 38110.

${ }^{2}$ Tecnológico Nacional de México, Instituto Tecnológico de Victoria, Boulevard Emilio Portes Gil \# 1301 Poniente, Ciudad Victoria, Tamaulipas, México. C.P. 87010.

* Autor para correspondencia E-mail: frcervantes@itroque.edu.mx

\section{RESUMEN}

El conocimiento del tipo de acción génica que controla los caracteres agronómicos es básico para la planeación y desarrollo de un programa de mejoramiento genético de maíz. El objetivo de este trabajo fue determinar la aptitud combinatoria general (ACG), aptitud combinatoria específica (ACE) y los efectos recíprocos (ER) involucrados en la expresión de caracteres agronómicos de líneas $\mathrm{S}_{3}$ de maíz. Las líneas y sus cruzas $F_{1}$ fueron evaluadas en dos fechas de siembra: 18 de marzo y 28 de mayo de 2014, donde se registraron los siguientes caracteres agronómicos: altura de planta, días a floración masculina y femenina, días a madurez fisiológica, aspecto general de planta, rendimiento de campo y algunos de sus componentes. Se encontró significancia entre genotipos y entre fechas de siembra para todos los caracteres evaluados. Con relación a los efectos de ACG y ACE, se encontraron diferencias significativas para todos los caracteres, con predominio de ACG sobre ACE para días a floración masculina y femenina, días a madurez fisiológica, altura de planta y componentes de rendimiento, pero en aspecto general de planta y rendimiento de campo el componente más importante fue la ACE. Los ER fueron significativos en altura de planta, días a floración femenina y aspecto general de planta. Las líneas TNM-5 y TNM-4 mostraron alta ACG en rendimiento y sus componentes y mayor precocidad. En las cruzas con estimadores altos y positivos en todas las características registradas, intervienen líneas con estimadores altos de ACG y con efectos bajos y negativos.

Palabras clave: Zea mays, cruzas dialélicas, efectos genéticos.

\section{ABSTRACT}

Knowing the type of gene action that controls agronomic traits is essential for the planning and development of a maize genetic improvement program. The objective of this study was to determine the genetic components of general combining ability (GCA), specific combining ability (SCA) and reciprocal effects (ER) involved in the expression of agronomic traits in a set of $S_{3}$ maize lines. $F_{1}$ crosses and their progenitors were evaluated at two planting dates: March 18 and May 28, 2014. The following agronomic traits were recorded: plant height, days to male and female flowering, days to physiological maturity, general appearance of the plant, crop yield and some of its components. 
Statistical differences were found between genotypes for all the traits in the two planting dates. Regarding the effects of GCA and SCA, significant differences were found for all the traits, with a predominance of GCA on SCA for days to male and female flowering, days to physiological maturity, plant height and yield. However, the most important component regarding general appearance of the plant and crop yield was SCA. The ER were significant in plant height, days to female flowering and general appearance of the plant. TNM-5 and TNM-4 lines showed high GCA in yield and its components, and greater precocity. In crosses with high and positive estimators in all the traits evaluated, lines with high ACG estimators and with low and negative effects intervene.

Key words: Zea mays, diallel crosses, genetic effects.

\section{INTRODUCCIÓN}

La viabilidad de la agricultura es fundamental para garantizar alimentos a la población. En este contexto, la seguridad alimentaria depende de varios factores, entre los que destacan el mantenimiento y fortalecimiento de un sistema de investigación y desarrollo de una manera incluyente, que sea capaz de proporcionar continuas mejoras en todos los aspectos de los sistemas productivos utilizados por los agricultores, que haga frente a la diversidad agroecológica, económica y social de un país, y que sea dinámica para responder a los cambios climáticos (Tansey y Rajotte, 2009). En este sentido, la FAO (2010) establece que a fin de alimentar a la población y mantener la producción agrícola, es condición indispensable disponer de suficientes semillas de calidad de variedades mejoradas de maíz. Al respecto, si el enfoque es la hibridación, Vergara et al. (2005) recomiendan conocer el comportamiento genético de las características de importancia económica de cada población, para elegir la estrategia de selección que permita obtener híbridos con mayores ventajas agronómicas. Asimismo, el mejorador requiere ampliar la base genética del germoplasma y evaluarla para tener conocimiento amplio del tipo de acción génica de los materiales en estudio para los caracteres de mayor importancia económica, tales como el rendimiento. Lo anterior permitirá elegir el mejor esquema de mejoramiento que maximice la varianza genética y de esta forma incrementar y fijar la frecuencia de genes favorables en la población (Luna et al., 2013; Haochuan et al., 2014).

Sprague y Tatum (1942) definieron la aptitud combinatoria general (ACG) como el comportamiento promedio de una línea en sus combinaciones híbridas, y la aptitud combinatoria específica (ACE) como los casos en los cuales ciertas combinaciones híbridas específicas se expresan favorablemente o no con respecto al comportamiento promedio de sus progenitores. En este contexto, Griffing (1956) desarrolló cuatro métodos para evaluar ACG y ACE considerando: 1) los progenitores y sus cruzas $\mathrm{F}_{1}$ directas y recíprocas; 2 ) los progenitores y sus cruzas directas $\mathrm{F}_{1 ;}$ ) las cruzas $\mathrm{F}_{1}$ directas y recíprocas; 4) la evaluación de las cruzas $\mathrm{F}_{1}$ directas únicamente.

Los análisis dialélicos constituyen una herramienta útil para estimar los parámetros genéticos de los progenitores y sus cruzas mediante ACG y ACE que permiten definir el método de mejoramiento genético más adecuado (Wong et al., 2007) y la evaluación y selección de líneas en generaciones tempranas $\left(S_{3}\right)$ y/o generaciones avanzadas $\left(\mathrm{S}_{10}\right)$ que permiten encontrar la mejor combinación hibrida para explotar la heterosis. Generalmente, líneas con alta ACG en pruebas tempranas conservan su postura en generaciones avanzadas.

Algunas investigaciones realizadas por Escorcia-Gutiérrez et al. (2010) y Guerrero-Guerrero et al. (2011) señalan que una cruza simple es de alto rendimiento cuando las dos líneas progenitoras son de alta ACG, o bien, al menos una línea es de alta ACG, pero presenta efectos positivos de ACE. Las líneas con efectos altos de ACG pueden emplearse para desarrollar variedades sintéticas, mientras que combinaciones específicas de alto rendimiento se pueden usar en la hibridación (Sprague y Eberhart, 1977). En este sentido, los efectos de ACG se relacionan con efectos aditivos que se pueden aprovechar por selección y luego por hibridación; mientras que los efectos de ACE se deben a efectos de dominancia y epistasis, y son utilizados por la hibridación en cruzas de materiales que combinan bien (Cervantes-Ortiz et al., 2016).

Por otro lado, otras investigaciones afirman una baja consistencia entre las líneas con estimadores de ACG altos y las combinaciones de ACE (Cervantes-Ortiz et al., 2007; Moterle et al., 2013). Algunos estudios genéticos realizados para caracteres fisiológicos, agronómicos y de rendimiento, han reportado diferencias estadísticas significativas entre las cruzas formadas (Dzib-Aguilar et al., 2011; Guerrero et al., 2011; Khalid et al., 2012; Adel y Ali, 2013; Conrado et al., 2014; Pérez-López et al., 2014; Hernández-Esparza et al., 2015) como resultado de la variabilidad genética entre las poblaciones de donde se derivan las lí- 
neas progenitoras, esto indica la posibilidad de obtener híbridos cada vez más rendidores, según indican Escorcia-Gutiérrez et al. (2010), lo que resalta la importancia de la variabilidad y la divergencia genética entre los progenitores de los híbridos.

Del mismo modo, se han reportado efectos significativos en ACG, ACE y efectos recíprocos (ER) en rendimiento de grano, días a floración y altura de planta, donde fueron mayores los efectos de ACG (Vergara et al., 2005; Cervantes-Ortiz et al., 2007; Dzib-Aguilar et al., 2011; Moterle et al., 2013; Conrado et al., 2014); sin embargo, otros autores destacan la importancia de los efectos de ACE (Guerrero et al., 2011; Badu-Apraku et al., 2013; Hussain y Ali, 2014). Lo anterior resalta la importancia de los efectos aditivos y de dominancia en la expresión de la variación genética para caracteres agronómicos.

Los efectos recíprocos (ER) son un factor relevante, por lo que pueden tener implicaciones en el mejoramiento genético del maíz (Hansen y Bagget, 1977). Por otro lado, la diversidad genética de los progenitores es un aspecto importante que influye en la expresión de los ER en varios caracteres del maíz (Kalsy y Sharma, 1972; Khehra y Bhalla, 1976). Tradicionalmente, en estudios de cruzas de maíz se hace énfasis sólo en ACG y $\mathrm{ACE}$ y se ignoran los efectos maternos. Al respecto, Dhliwayo et al. (2005) y Haro et al. (2007) encontraron diferencias en la expresión fenotípica de las cruzas en maíz y girasol en características como acame de tallo, resistencia a plagas, rendimiento de grano y en calidad física y química de la semilla, debido a que los patrones de herencia están controlados por efectos maternos y paternos.

En base a lo anterior, el objetivo de esta investigación fue determinar los parámetros genéticos de ACG, ACE y ER involucrados en la expresión de caracteres agronómicos de líneas $\mathrm{S}_{3}$ de maíz y en sus cruzas directas y recíprocas.

\section{MATERIALES Y MÉTODOS}

En este trabajo se utilizaron cinco líneas de maíces blancos con nivel de endogamia $\mathrm{S}_{3}$ derivadas del programa de mejoramiento del Instituto Tecnológico de Roque (ITR) (Tabla 1), las cuales fueron cruzadas de forma directa y recíproca para generar un dialélico completo de 20 combinaciones bajo el método I de Griffing (1956).

Los cruzamientos se realizaron durante el ciclo Primavera-Verano 2013, en el campo experimental del ITR, donde al mismo tiempo se incrementaron las líneas. Se formaron 25 genotipos: 10 cruzas directas, 10 cruzas recíprocas y 5 progenitores, los cuales fueron cosechados por separado en Octubre de 2013.

Las cruzas $\mathrm{F}_{1}$ directas y recíprocas y sus progenitores fueron evaluados en el campo experimental del ITR, ubicado en Celaya, Guanajuato, México, que de acuerdo con Google Earth ${ }^{\circledR}$ se localiza a los $20^{\circ} 32^{\prime} 05^{\prime \prime} \mathrm{N}$ y $100^{\circ} 50^{\prime} 00^{\prime \prime} \mathrm{O}$ a una altitud de $1752 \mathrm{msnm}$. El clima de esta región es semi cálido, con una precipitación anual entre los 400 a $700 \mathrm{~mm}$. El régimen térmico es de 18 a $20^{\circ} \mathrm{C}$. Los suelos son del tipo vertisol pélico (Mora et al., 2001).

La siembra se realizó en dos fechas: 18 de marzo y 28 de mayo de 2014. En ambas fechas se empleó un diseño de bloques completos al azar con tres repeticiones. La unidad experimental consistió de dos surcos de 0,75 m de separación y $5 \mathrm{~m}$ de largo. En ambas fechas, la siembra se realizó en seco y fue manual con depósito de una semilla cada $16 \mathrm{~cm}$, lo que originó 80.000 plantas ha $^{-1}$. Se aplicó la dosis de fertilización 240-60-60: $50 \%$ del nitrógeno, todo el fósforo y potasio se aplicaron al momento de la siembra; la otra mitad de nitrógeno se aplicó 40 días después de la siembra (dds). El riego se aplicó por gravedad a la parcela en tubos de PVC de compuertas. En la primera fecha de siembra se aplicaron cuatro riegos de auxilio y en la segunda tres. El control

Tabla 1. Origen y características agronómicas de líneas $\mathrm{S}_{3}$ de maíz utilizadas en el diseño I de cruzas dialélicas de Griffing.

Table 1. Origin and agronomic traits of $\mathrm{S}_{3}$ maize lines used in a diallel cross design I of Griffing.

\begin{tabular}{|c|c|c|c|c|}
\hline Línea & $\begin{array}{l}\text { Nivel de } \\
\text { endogamia }\end{array}$ & Origen de las líneas & Origen del híbrido & Color de grano \\
\hline TNM-1 & $\mathrm{S}_{3}$ & Pob. $F_{2}(D K 2042)$ & Monsanto & Blanco \\
\hline TNM-2 & $\mathrm{S}_{3}$ & Pob. F $F_{2}$ (DK2027) & Monsanto & Blanco \\
\hline TNM-3 & $\mathrm{S}_{3}$ & Pob. $F_{2}(\mathrm{P} 3030 \mathrm{~W})$ & Pioneer & Blanco \\
\hline TNM-4 & $S_{3}^{3}$ & Pob. F (Caimán) & Monsanto & Blanco \\
\hline TNM-5 & $\mathrm{S}_{3}^{3}$ & Variedad Roque & ITR & Blanco \\
\hline
\end{tabular}

Pob = Población; ITR = Instituto Tecnológico de Roque. 
de malezas se hizo con $4 \mathrm{~L} \mathrm{ha}^{-1}$ de Atrazina (25\%) y Metolachlor (25\%) en etapa V6, y mediante dos escardas mecánicas (20 y 40 dds). En etapas tempranas del cultivo se controló la presencia de trips con 0,5 L ha-1 de Dimetoato; posteriormente, se aplicó $1 \mathrm{~L} \mathrm{ha}^{-1}$ de Cipermetrina para controlar gusano cogollero (Spodoptera frugiperda).

En las dos fechas de siembra se midió: la altura de planta (AP), días a floración masculina (DFM) y femenina (DFF), días a madurez fisiológica (DMF) y apariencia general de planta (AGP).

La AP se determinó tomando tres plantas al azar de la parcela; los DFM y DFF se determinaron considerando los días transcurridos entre el primer riego y la fecha en que el $50+1 \%$ de las plantas de cada unidad experimental mostraron los estigmas entre 2 y $3 \mathrm{~cm}$ de largo y anteras abiertas liberando polen, respectivamente; los DMF se obtuvieron contabilizando el número de días transcurridos del primer riego hasta el momento en que el grano estaba en estado lechoso; y la AGP se registró de forma visual después de madurez fisiológica cuando la planta presentó uniformidad de follaje seco y el grano se encontraba en etapa de secado (antes de cosecha). La evaluación se hizo usando la escala del 1 al 9, donde: 1 es deficiente y 9 es excelente.

Por otro lado, el rendimiento de grano en campo (RC) se obtuvo al cosechar los dos surcos de la parcela útil, eliminando un metro en cada extremo. Además, en cinco mazorcas tomadas al azar de cada unidad experimental se determinaron el diámetro de mazorca (DM) y longitud de mazorca (LM); en grano se determinó el peso de
100 semillas (p100s), considerando cuatro repeticiones de 100 semillas por unidad experimental.

Para los datos de cada variable se realizó un análisis de varianza para determinar la aptitud combinatoria general (ACG), aptitud combinatoria específica (ACE) y efectos recíprocos (ER), de acuerdo con el método I de Griffing (1956). Se usó el programa de cómputo Diallel-SAS method I (Zhang y Kang, 2003). El modelo lineal general es:

$Y i j k=\mu+g_{i}+g_{j}+s_{i j}+l_{k}+(g l)_{i k}+(g l)_{j k}+(s l)_{i j k}+\varepsilon_{i j k}$ donde, $Y_{i j k}=$ valor observado de cada unidad experimental en el ambiente $k$ de los progenitores i y j; $\mu=$ media general; $g_{i}$ o $g=$ efectos de ACG de los progenitores; sij= efectos de ACE de cruzas entre los padres i y j; $l_{k}=$ efecto del ambiente $\mathrm{k}$; $(g l)_{i k}=$ efectos de interacción de ACG de los progenitores i o j con el ambiente $\mathrm{k} ;(s l)_{i j k}=$ efectos de interacción de ACE de las cruzas ij y el ambiente $\mathrm{k} ; \mathrm{y} \varepsilon_{i j k}=$ efecto residual.

\section{RESULTADOS Y DISCUSIÓN}

El análisis de varianza detectó diferencias estadísticas $(P \leq 0,01)$ entre genotipos (cruzas y progenitores) para todas las variables (Tabla 2) evaluadas en las dos fechas de siembra (FS). Entre fechas de siembra hubo diferencias estadísticas ( $P$ $\leq 0,01)$ para todos los caracteres agronómicos, excepto para AGP que tuvo efecto significativo de $\mathrm{P}$ $\leq 0,05$. La interacción genotipo por fecha de siembra $(\mathrm{G} \times \mathrm{FS})$ resultó significativa $(\mathrm{P} \leq 0,01)$ para DMF y AGP y fue significativa a $\mathrm{P} \leq 0,05$ para DFF. La interacción aptitud combinatoria específica por fecha de siembra (ACE x FS) fue signi-

Tabla 2. Cuadrados medios del análisis dialélico de cinco líneas $S_{3}$ de maíz blanco para cinco caracteres agronómicos.

Table 2. Mean squares of diallel analysis of five $S_{3}$ lines of white maize for five agronomic traits.

\begin{tabular}{lrlcccc}
\hline F.V. & G.L. & $\begin{array}{l}\text { AP } \\
(\mathbf{m})\end{array}$ & DFF & $\begin{array}{c}\text { DFM } \\
\text { (días) }\end{array}$ & DMF & $\begin{array}{c}\text { AGP } \\
(\mathbf{\%})\end{array}$ \\
\hline Genotipos (G) & 24 & $0,17^{* *}$ & $33,27^{* *}$ & $34,83^{* *}$ & $33,60^{* *}$ & $2,85^{* *}$ \\
ACG & 4 & $0,36^{* *}$ & $57,29^{* *}$ & $59,94^{* *}$ & $56,94^{* *}$ & $3,50^{* *}$ \\
ACE & 10 & $0,22^{* *}$ & $30,59^{* *}$ & $30,79^{* *}$ & $27,22^{* *}$ & $4,64^{* *}$ \\
ER & 10 & $0,05^{*}$ & $7,28^{*}$ & 7,93 & 5,27 & $0,58^{*}$ \\
Fecha de siembra(FS) & 1 & $1,68^{* *}$ & $816,66^{* *}$ & $927,52^{* *}$ & $1700,16^{* *}$ & $1,04^{*}$ \\
G*F & 24 & 0,02 & $4,09^{*}$ & 3,80 & $6,09^{* *}$ & $0,98^{* *}$ \\
ACG*FS & 4 & 0,03 & 8,25 & 8,79 & 7,75 & 0,85 \\
ACE*FS & 10 & 0,01 & 3,93 & 3,64 & $10,35^{* *}$ & $1,34^{* *}$ \\
ER*FS & 10 & 0,02 & 4,76 & 4,51 & 5,12 & $0,66^{* *}$ \\
Error & 96 & 0,02 & 2,76 & 2,77 & 2,91 & 0,22 \\
C.V. $(\%)$ & ---- & 7,27 & 2,30 & 2,24 & 1,65 & 5,40 \\
\hline
\end{tabular}

* ** Diferente de cero a una probabilidad de 0,05 y 0,01, respectivamente. F.V.= fuente de variación, G.L.= grados de libertad. AP, DFF, DFM, DMF y AGP; corresponden a la altura de planta, días a floración femenina, días a floración masculina, días a madurez fisiológica y aspecto general de planta, respectivamente. 
ficativa $(\mathrm{P} \leq 0,01)$ para DMF y AGP. Finalmente, la interacción de efectos recíprocos por fecha de siembra (ER x FS) fue significativa únicamente para AGP.

Con relación a los efectos de ACG y ACE se encontraron diferencias significativas para todos los caracteres, mostrando que hubo variabilidad en efectos genéticos aditivos y dominantes. La proporción relativa de los efectos de ACG y de ACE determinados por los cuadrados medios indica el tipo de acción génica en los caracteres (El-Badawy, 2013). En este sentido, los cuadrados medios de los efectos de ACG fueron mayores que los de ACE para todos los caracteres, excepto para AGP (Tabla 2), lo que resalta la importancia de los efectos aditivos sobre los no aditivos en estos caracteres (Pswarayi y Vivek, 2008; De la Cruz et al., 2010; Zare et al., 2011; Umar et al., 2014; Andrio-Enríquez et al., 2015). Por el contrario, Hussain y Ali (2014) destacan a los efectos genéticos no aditivos como el componente más importante en caracteres agronómicos en maíz, cuando se habla de hibridación.

Los efectos recíprocos (ER) fueron significativos para AP, DFF y AGP, lo que indica que estos caracteres son afectados por el progenitor femenino utilizado en la producción de semilla; estos resultados coinciden con los reportados por Cervantes-Ortiz et al. (2007); Zare et al. (2011) y Silva et al. (2015) en trabajos similares en maíz.

Con respecto al rendimiento de campo y sus componentes, se detectaron diferencias estadísticas $(P \leq 0,01)$ entre genotipos (cruzas y progenitores) para todos los caracteres (Tabla 3). Esto puede atribuirse a la diversidad genética entre los progenitores por su origen (Tabla 1). En este sentido, Guillen-De la Cruz et al. (2009) mencionan que a medida que se incrementa la diversidad genética entre los progenitores, hasta un límite, se incrementan las diferencias entre sus cruzas para caracteres agronómicos y fisiológicos.

Al descomponer la fuente de variación de genotipos en los efectos de ACG y ACE se detectaron diferencias significativas $(\mathrm{P} \leq 0,01)$ para todas las características, lo que difiere de lo reportado por Ojo et al. (2007), quienes afirman que los efectos de ACG y ACE no son significativos en la expresión de los componentes del rendimiento en maíz.

Los efectos genéticos aditivos (ACG) fueron de mayor expresión en LM, DM y p100s, y los efectos no aditivos (dominancia) fueron el componente más importante para RC (Tabla 3). Estos resultados concuerdan con los reportados por De la Cruz et al. (2010), Aminu y Izge (2013), Badu-Apraku et al. (2013) y Aminu et al. (2014), quienes encontraron que la acción genética no aditiva constituye el componente genético más importante del rendimiento de grano en maíz; pero difieren de los reportados por Ojo et al. (2007), Umar et al. (2014) y Antuna et al. (2017), quienes afirman que en el rendimiento de grano los efectos de ACG son el componente más importante. Al respecto, Cervantes-Ortiz et al. (2016) mencionan que cuando en una población los efectos de acción genética aditiva son más importantes que los de acción genética no aditiva, se recomienda mejorar la población por selección recurrente para seguir acumulando genes o frecuencias génicas deseables; por el contrario, si los efectos de acción no aditiva (dominancia) son más importantes, la población debe mejorarse por hibridación.

La interacción genotipo por fecha de siembra (G x FS) y de aptitud combinatoria general por fecha de siembra (ACG $\times$ FS) fueron significativas $(\mathrm{P} \leq 0,05)$ solo para $\mathrm{p} 100 \mathrm{~s}$; la aptitud combinatoria específica por fecha de siembra (ACE x FS) fue significante $(\mathrm{P} \leq 0,05)$ únicamente para LM. Finalmente, entre fechas de siembra (FS) hubo significancia a $\mathrm{P} \leq 0,05$ para el $R C$, $\mathrm{LM}$ y con $\mathrm{P} \leq 0,01$ para p100s (Tabla 3).

La significancia de la interacción ACG x FS indica la importancia de los efectos aditivos en el p100s, por lo que los efectos de ACG variaron con la fecha de siembra y entonces se debe seleccionar a los progenitores con efectos altos de ACG a través de fechas de siembra. Por otro lado, la interacción ACE x FS indica que las cruzas no mantuvieron su efecto de ACE para DMF, AGP y LM en las fechas de siembra. Esto puede atribuirse a la variación ambiental presentada en cada fecha de siembra y al comportamiento diferencial de progenitores (De la Cruz et al., 2010; Mhike et al., 2011; Esquivel et al., 2013).

Los coeficientes de variación tuvieron valores bajos en la mayoría de los caracteres registrados, por lo que se tiene alta confiabilidad en los datos y por tanto en los resultados.

De acuerdo con los estimadores de los efectos de ACG (Tabla 4), las líneas TNM-5 y TNM-4 presentaron valores positivos altos para caracteres agronómicos y estimadores negativos en precocidad; de tal forma que estas dos líneas alcanzaron la madurez fisiológica a los 103 días y un rendimiento promedio de 4,4 y 4,0 $\mathrm{t} \mathrm{ha}^{-1}$, respectivamente (Tabla 5). Por el contrario, la línea TNM-2 presentó el peor comportamiento para caracteres de rendimiento (estimadores negativos) con rendimiento de 1,5 $\mathrm{t} \mathrm{ha}^{-1} \mathrm{y}$ además fue el genotipo más tardío con 112,3 días a madurez fisiológica (Tabla 5). Estos resultados indican que las líneas TNM-5 y TNM-4 tienen una buena contribución en caracteres agronómicos y en la expresión del rendimiento. La identificación de líneas en pruebas tempranas con propósitos de utilización en 
Tabla 3. Cuadrados medios del análisis dialélico de cinco líneas $S_{3}$ de maíz para rendimiento y sus componentes.

Table 3. Mean squares of diallel analysis of five $S_{3}$ maize lines for yield and its components.

\begin{tabular}{lccccc}
\hline F.V. & G.L. & $\begin{array}{c}\text { RC } \\
\left(\mathbf{t ~ h a}^{-1}\right)\end{array}$ & $\begin{array}{c}\text { LM } \\
(\mathbf{c m})\end{array}$ & $\begin{array}{c}\text { DM } \\
(\mathbf{m m})\end{array}$ & $\begin{array}{c}\text { p100s } \\
\text { (g) }\end{array}$ \\
\hline Genotipos (G) & 24 & $23,12^{* *}$ & $8,60^{* *}$ & $48,38^{* *}$ & $1452,72^{* *}$ \\
ACG & 4 & $14,05^{* *}$ & $20,28^{* *}$ & $103,33^{* *}$ & $6455,11^{* *}$ \\
ACE & 10 & $42,50^{* *}$ & $9,53^{* *}$ & $72,46^{* *}$ & $671,02^{* *}$ \\
ER & 10 & 2,39 & 3,01 & 2,31 & 168,66 \\
Fecha de siembra (FS) & 1 & $8,37^{*}$ & $86,23^{* *}$ & 0,62 & $3778,02^{* *}$ \\
G FS $_{\text {ACG FS }}^{24}$ & 2,10 & 3,62 & 10,73 & $168,59^{*}$ \\
ACE*FS & 4 & 3,14 & 2,34 & 12,93 & $245,15^{*}$ \\
ER*FS & 10 & 2,69 & $5,75^{*}$ & 18,84 & 182,01 \\
Error & 10 & 1,28 & 2,01 & 1,75 & 124,55 \\
C.V. $(\%)$ & 96 & 1,75 & 2,86 & 11,35 & 110,77 \\
\hline
\end{tabular}

*,** Diferente de cero a una probabilidad de 0,05 y 0,01, respectivamente. F.V.= fuente de variación, G.L.= grados de libertad. RC, LM, DM y p100s; corresponden a rendimiento de campo, longitud de mazorca, diámetro de mazorca y peso de cien semillas, respectivamente.

Tabla 4. Efectos de aptitud combinatoria general (ACG) para caracteres agronómicos en cinco líneas $\mathrm{S}_{3}$ de maíz.

Table 4. Effects of general combining ability (GCA) for agronomic traits in five $\mathrm{S}_{3}$ corn lines.

\begin{tabular}{llllllllll}
\hline Líneas & $\begin{array}{c}\text { AP } \\
(\mathbf{m})\end{array}$ & DFF & $\begin{array}{c}\text { DFM } \\
\text { (días) }\end{array}$ & DMF & $\begin{array}{c}\text { AGP } \\
(\mathbf{\%})\end{array}$ & $\begin{array}{c}\text { RC } \\
(\mathbf{t ~ h a - 1})\end{array}$ & $\begin{array}{c}\text { LM } \\
(\mathbf{c m})\end{array}$ & $\begin{array}{c}\text { DM } \\
(\mathbf{m m})\end{array}$ & $\begin{array}{c}\text { p100s } \\
(\mathbf{g})\end{array}$ \\
\hline TNM-1 & $-0,09^{* *}$ & $1,31^{* *}$ & $1,35^{* *}$ & $1,26^{* *}$ & 0,03 & $-0,41$ & 0,18 & $-0,33$ & $-9,58^{* *}$ \\
TNM-2 & 0,02 & 0,26 & 0,33 & $0,53^{*}$ & $-0,39^{* *}$ & $-0,29$ & $-0,88^{* *}$ & $-1,49^{* *}$ & $-7,48^{* *}$ \\
TNM-3 & $0,05^{* *}$ & 0,26 & 0,22 & 0,19 & $-0,02$ & $-0,09$ & 0,27 & $-0,88^{*}$ & $-2,32^{*}$ \\
TNM-4 & 0,02 & $-0,30$ & $-0,38$ & $-0,59^{*}$ & $0,15^{* *}$ & $-0,03$ & $-0,21$ & $1,14^{* *}$ & $3,02^{*}$ \\
TNM-5 & $0,10^{* *}$ & $-1,53^{* *}$ & $-1,53^{* *}$ & $-1,39^{* *}$ & $0,23^{* *}$ & $0,84^{* *}$ & $0,64^{* *}$ & $1,57^{* *}$ & $16,36^{* *}$ \\
\hline
\end{tabular}

*,** Diferente de cero a una probabilidad de 0,05 y 0,01, respectivamente. AP, DFF, DFM, DMF, AGP, RC, LM, DM y p100s; corresponden a la altura de planta, días a floración femenina, días a floración masculina, días a madurez fisiológica, aspecto general de planta, rendimiento de campo, longitud de mazorca, diámetro de mazorca y peso de cien semillas, respectivamente.

programas de mejoramiento genético, debe tomar en cuenta los efectos de ACG y ACE, dependiendo de los objetivos. Por lo tanto, las líneas TNM4 y TNM5, con base a los mejores efectos de ACG pueden incluirse para formar variedades sintéticas y las cruzas con los mejores efectos de ACE pueden formar parte de un programa de mejoramiento para formación de híbridos (Guillen-De la Cruz et al., 2009; De la Cruz et al., 2010).

En las cruzas el efecto $S_{i j}$ promedio para las mejores cuatro cruzas en rendimiento (TNM$2 \times$ TNM-4, TNM-2 $\times$ TNM-3, TNM-1 $\times$ TNM-5 y TNM-2 x TNM-5) fueron de 2,08, 2,04, 1,09 y 0,98 , respectivamente, con rendimiento de campo promedio de $14,2,12,6,13,1$ y $13,2 \mathrm{t} \mathrm{ha}^{-1}$, respectivamente (Tabla 6 y 7). Con base en los estimado- res (efectos genéticos) de las cruzas (Tabla 6) se presentaron efectos altos y positivos para rendimiento de campo; sin embargo, en estas cruzas intervienen tanto líneas con alta ACG, como con valores bajos y negativos (Tabla 4). En este sentido, se esperaba que las cruzas de mayor rendimiento hubieran sido el resultado de cruzar dos líneas de altos efectos positivos de ACG. Sin embargo, no resultó así en este estudio, lo cual sugiere que no necesariamente las líneas con alta ACG pueden producir cruzas sobresalientes en rendimiento de grano. Resultados similares fueron obtenidos por Guillen-De la Cruz et al. (2009) al cruzar germoplasma de efectos negativos de ACG y obtener valores altos y positivos de ACE de cruzas. Por otro lado, Escorcia-Gutiérrez et al. (2010) 
Tabla 5. Valores promedio para caracteres agronómicos en líneas de maíz.

Table 5. Mean values for agronomic traits in corn lines.

\begin{tabular}{cccccccccc}
\hline Líneas & $\begin{array}{c}\text { AP } \\
\text { (m) }\end{array}$ & DFF & $\begin{array}{c}\text { DFM } \\
\text { (días) }\end{array}$ & DMF & $\begin{array}{c}\text { AGP } \\
\text { (\%) }\end{array}$ & $\begin{array}{c}\text { RC } \\
\left(\mathbf{t ~ h a}^{-1}\right)\end{array}$ & $\begin{array}{c}\text { LM } \\
\text { (cm) }\end{array}$ & $\begin{array}{c}\text { DM } \\
\text { (cm) }\end{array}$ & $\begin{array}{c}\text { p100s } \\
\text { (g) }\end{array}$ \\
\hline TNM-1 & 1,53 & 80,8 & 83,0 & 106.3 & 80,1 & 3,2 & 17,0 & 4,9 & 31,6 \\
TNM-2 & 1,70 & 73,8 & 76,0 & 112.3 & 60,3 & 1,5 & 14,9 & 4,8 & 30,9 \\
TNM-3 & 1,85 & 74,5 & 76,3 & 106.6 & 70,7 & 3,8 & 16,6 & 4,5 & 31,4 \\
TNM-4 & 1,81 & 74,5 & 76,6 & 103.3 & 80,0 & 4,0 & 16,6 & 5,0 & 34,3 \\
TNM-5 & 2,18 & 72,3 & 74,5 & 103.3 & 80,1 & 4,4 & 18,0 & 5,3 & 34,4 \\
\hline
\end{tabular}

AP, DFF, DFM, DMF, AGP, RC, LM, DM y p100s; corresponden a la altura de planta, días a floración femenina, días a floración masculina, días a madurez fisiológica, aspecto general de planta, rendimiento de campo, longitud de mazorca, diámetro de mazorca y peso de cien semillas, respectivamente.

Tabla 6. Efectos de aptitud combinatoria específica (ACE) de 10 cruzas directas y 10 cruzas recíprocas de maíz para caracteres agronómicos.

Table 6. Effects of specific combining ability (ACE) of 10 direct crosses and 10 reciprocal crosses of maize for agronomic traits.

\begin{tabular}{|c|c|c|c|c|c|c|c|c|c|}
\hline Cruzas & $\begin{array}{c}\text { AP } \\
(\mathrm{cm})\end{array}$ & DFF & $\begin{array}{l}\text { DFM } \\
\text { (días) }\end{array}$ & DMF & $\begin{array}{c}\text { AGP } \\
(\%)\end{array}$ & $\begin{array}{c}\mathrm{RC} \\
\left(\mathrm{t} \mathrm{ha} \mathbf{a}^{-1}\right)\end{array}$ & $\begin{array}{c}\mathrm{LM} \\
(\mathrm{cm})\end{array}$ & $\begin{array}{c}\mathrm{DM} \\
(\mathrm{mm})\end{array}$ & $\begin{array}{c}\text { p100s } \\
\text { (g) }\end{array}$ \\
\hline & \multicolumn{9}{|c|}{ Cruzas directas } \\
\hline TNM-1 X TNM-2 & $0,09^{* *}$ & $-0,68$ & $-0,72$ & $-0,63$ & $0,42^{* *}$ & 0,42 & 0,15 & $1,64^{*}$ & $-1,21$ \\
\hline TNM-1 X TNM-3 & $0,07^{*}$ & $-0,84^{*}$ & $-0,82^{*}$ & $1,74^{* *}$ & $0,20^{*}$ & 0,32 & 0,64 & $4^{*}$ & $7,97^{* *}$ \\
\hline TNM-1 X TNM-4 & $-0,01$ & $-2,11^{* *}$ & $-1,77^{* *}$ & $-1,3$ & 0,17 & 0,08 & $-0,01$ &, 26 & 3,00 \\
\hline TNM-1 X TNM-5 & $0.08^{*}$ & $-1,37^{* *}$ & $-1,54^{* *}$ & $-1,79^{* *}$ & $-0,05$ & $1,09^{* *}$ & $0,69^{*}$ & 0,04 & $7,97^{* *}$ \\
\hline TNM-2 X TNM-3 & 0,04 & 0,20 & 0,13 & 0,33 & 0,16 & $2,04^{* *}$ & 0,27 & $04^{*}$ & $-2,41$ \\
\hline TNM-2 X TNM-4 & $0,12^{* *}$ & $-0,23$ & $-0,41$ & $-0,12$ & $0,49^{* *}$ & 2,0 & $0,71^{*}$ & $1,99^{*}$ & $-0,53$ \\
\hline TNM-2 X TNM-5 & 0,03 & $-0,33$ & $-0,10$ & 0,26 & $0,52^{* *}$ & $0,98^{* *}$ & 0,27 & 0,14 & $-1,61$ \\
\hline TNM-3 X TNM-4 & 0,02 & $-0,22$ & $-0,14$ & $0,88^{*}$ & 0,19 & $0,63^{*}$ & 0,54 & 0,73 & $6,98^{* *}$ \\
\hline TNM-3 X TNM-5 & $-0,02$ & $-0,82^{*}$ & $-0,82^{*}$ & $-0,6$ & $0,40^{* *}$ & $-0,1$ & 0,57 & 0,71 & 0,80 \\
\hline \multirow[t]{2}{*}{ TNM-4 X TNM-5 } & $0,08^{*}$ & $-0,59$ & $-0,88^{*}$ & $-0,85^{*}$ & 0,12 & $-0,55$ & $-0,17$ & 1,21 & 1,50 \\
\hline & \multicolumn{9}{|c|}{ Cruzas recíprocas } \\
\hline TNM-2 X TNM-1 & $-0,09^{*}$ & 0,17 & 0,1 & $-0,10$ & 0,05 & 0,23 & $-0,90^{*}$ & 0,07 & $-0,71$ \\
\hline TNM-3 X TNM-1 & $-0,00$ & $-1,32^{*}$ & $-1,48^{* *}$ & $-0,8$ & $-0,20$ & $-0,06$ & $-0,41$ & $-0,24$ & $-8,77^{* *}$ \\
\hline TNM-4 X TNM-1 & $0,07^{*}$ & 0,33 & 0,41 & $-0,25$ & $-0,35^{*}$ & 0,44 & 0,48 & $-0,57$ & $-1,85$ \\
\hline TNM-5 X TNM-1 & 0,06 & 0,33 & 0,50 & 0,16 & $-0,20$ & 0,24 & 0,08 & 1,10 & $-2,83$ \\
\hline TNM-3 X TNM-2 & 0,06 & $1,33^{*}$ & $1,08^{*}$ & $1,50^{*}$ & $-0,16$ & $-0,32$ & 0,58 & 0,36 & $-3,06$ \\
\hline TNM-4 X TNM-2 & 0,00 & $-1,00^{*}$ & $-1,08^{*}$ & $-0,41$ & $-0,36^{*}$ & $0,76^{*}$ & $-0,51$ & $-0,23$ & $-3,31$ \\
\hline TNM-5 X TNM-2 & 0,06 & 0,66 & 0,7 & 0 & $-0,05$ & 0,26 & 0,40 & 0,13 & $-2,03$ \\
\hline TNM-4 X TNM-3 & $0,08^{*}$ & 0,66 & 0,58 & 0,75 & $-0,10$ & 0,02 & 0,41 & 0,23 & $-1,71$ \\
\hline TNM-5 X TNM-3 & 0,05 & $-0,16$ & $-0,08$ & 0,08 & $-0,08$ & $-0,68$ & 0,27 & 0,17 & 0,13 \\
\hline TNM-5 X TNM-4 & 0,06 & 0,50 & 0,75 & 0,75 & $0,29^{*}$ & 0,66 & 0,51 & $-0,15$ & 4,91 \\
\hline
\end{tabular}

* ** significativo al 0,05 y 0,01 de probabilidad, respectivamente. AP, FF, FM, MF, AGP, RC, LM, DM, y p100s; corresponden a la altura de planta, días a floración femenina, días a floración masculina, días a madurez fisiológica, aspecto general de planta, rendimiento de campo, longitud de mazorca, diámetro de mazorca y peso de cien semillas, respectivamente.

y Guerrero-Guerrero et al. (2011) mencionan que se espera que en las cruzas con mayor rendimiento (ACE) intervengan las dos líneas de alta ACG, o que al menos una línea sea de alta ACG. Cruzas similares fueron obtenidas por Cervantes-Ortiz et al. (2007) y De la Cruz et al. (2010). En este sentido, se establece que las cruzas con valores altos de ACE pueden considerarse en un programa de mejoramiento genético por hibridación.

Para DFF, DFM y DMF sobresalen las cruzas TNM-1 x TNM-4, TNM-1 x TNM-5, TNM-3 x TNM-1 y TNM-1 x TNM-3 con estimadores negativos y significativos, lo que indica que presentaron mayor precocidad (Tabla 6); por otro lado, 
Tabla 7. Comportamiento promedio de cruzas directas y recíprocas para caracteres agronómicos en maíz.

Table 7. Average behavior of direct and reciprocal crosses for agronomic traits in corn.

\begin{tabular}{|c|c|c|c|c|c|c|c|c|c|}
\hline Cruzas & $\begin{array}{l}\text { AP } \\
(\mathrm{m})\end{array}$ & DFF & $\begin{array}{l}\text { DFM } \\
\text { (días) }\end{array}$ & DMF & $\begin{array}{r}\text { AGP } \\
(\%)\end{array}$ & $\begin{array}{c}\text { RC } \\
\left(\mathbf{t} \mathbf{h a}^{1}\right)\end{array}$ & LM & $\begin{array}{l}\mathrm{DM} \\
\mathrm{Cm}\end{array}$ & $\begin{array}{l}\text { p100s } \\
\text { (g) }\end{array}$ \\
\hline \multicolumn{10}{|c|}{ Cruzas directas } \\
\hline TNM-1 X TNM-2 & 2,20 & 73,3 & 75,3 & 104,1 & 90,1 & 10,8 & 16,6 & 4,2 & 34,0 \\
\hline TNM-1 X TNM-3 & 2,21 & 70,0 & 71,1 & 100,0 & 90,0 & 10,5 & 16,8 & 4,2 & 38,7 \\
\hline TNM-1 X TNM-4 & 2,42 & 70,3 & 71,8 & 100,1 & 88,8 & 11,0 & 18,5 & 4,3 & 37,2 \\
\hline TNM-1 X TNM-5 & 2,65 & 70,0 & 71,0 & 101,3 & 88,5 & 13,1 & 19,7 & 4,4 & 39,0 \\
\hline TNM-2 X TNM-3 & 2,45 & 74,3 & 76,0 & 103,6 & 85,7 & 12,6 & 17,3 & 5,2 & 34,8 \\
\hline TNM-2 X TNM-4 & 2,56 & 71,0 & 72,6 & 102,5 & 86,7 & 14,2 & 19,2 & 5,4 & 45,0 \\
\hline TNM-2 X TNM-5 & 2,65 & 71,3 & 73,6 & 103,0 & 95,0 & 13,2 & 18,5 & 4,2 & 34,8 \\
\hline TNM-3 X TNM-4 & 2,54 & 72,6 & 74,5 & 104,3 & 94,1 & 11,6 & 19,1 & 4,3 & 37,7 \\
\hline TNM-3 X TNM-5 & 2,60 & 70,0 & 72,0 & 101,3 & 95,2 & 10,8 & 19,8 & 5,1 & 34,0 \\
\hline TNM-4 X TNM-5 & 2,32 & 70,0 & 72,1 & 100,8 & 95,2 & 12,9 & 18,8 & 5,0 & 33,6 \\
\hline \multicolumn{10}{|c|}{ Cruzas recíprocas } \\
\hline TNM-2 X TNM-1 & 2.0 & 72,0 & 74,0 & 103,3 & 90.5 & 11,2 & 18,4 & 5,1 & 33,4 \\
\hline TNM-3 X TNM-1 & 2,43 & 73,3 & 75,3 & 101,5 & 95.3 & 11,4 & 19,6 & 4,2 & 35,2 \\
\hline TNM-4 X TNM-1 & 2,63 & 70,8 & 73,0 & 102,6 & 95.4 & 9,9 & 17,5 & 4,4 & 34,8 \\
\hline TNM-5 X TNM-1 & 2,56 & 70,3 & 72,0 & 101,0 & 95.1 & 12,6 & 19,5 & 5,2 & 35,7 \\
\hline TNM-3 X TNM-2 & 2,70 & 71,6 & 73,8 & 102,6 & 88.6 & 13,5 & 17,1 & 5,1 & 34,2 \\
\hline TNM-4 X TNM-2 & 2,28 & 73,0 & 74,8 & 103,0 & 95.2 & 12,2 & 18,2 & 5,4 & 35,8 \\
\hline TNM-5 X TNM-2 & 2,35 & 70,0 & 72,1 & 102,0 & 95.5 & 12,5 & 17,7 & 4,8 & 35,7 \\
\hline TNM-4 X TNM-3 & 2,78 & 71,3 & 73,3 & 102,8 & 95.1 & 11,6 & 18,3 & 5,1 & 34,9 \\
\hline TNM-5 X TNM-3 & 2,90 & 70,3 & 72,1 & 101,1 & 95.4 & 12,6 & 19,3 & 5,1 & 35,5 \\
\hline TNM-5 X TNM-4 & 2,94 & 69,3 & 70,6 & 102,6 & 95.4 & 10,4 & 17,8 & 5,0 & 36,8 \\
\hline
\end{tabular}

AP, FF, FM, MF, AGP, RC, LM, DM, y p100s; corresponden a la altura de planta, días a floración femenina, días a floración masculina, días a madurez fisiológica, aspecto general de planta, rendimiento de campo, longitud de mazorca, diámetro de mazorca y peso de cien semillas, respectivamente.

cuando son comparado estos estimadores con las medias, se observa efectivamente que dichas cruzas fueron las más precoces ya que alcanzaron la madurez fisiológica en sólo 100 días. Esta característica es importante en aquellos ambientes donde se presentan heladas tempranas o falta de lluvias; o bien, donde se tiene necesidad de desocupar el terreno agrícola para las labores de preparación de la siembra siguiente, por lo que es importante considerar estas cruzas en un programa de mejoramiento genético para precocidad. Así mismo, la cruza TNM-2 x TNM-1 presentó estimadores negativos y valores bajos $(2,0 \mathrm{~m})$ en $\mathrm{AP}$. $\mathrm{Al}$ respecto, se considera deseable contar con materiales de porte bajo, ya que estos toleran altas densidades de plantas y resisten al acame. Finalmente, las cruzas TNM-1 x TNM-5 y TNM-2 x TNM-4 destacan con estimadores altos y positivos para LM, en DM sobresalieron las cruzas TNM- 2 TNM-3 y TNM-2 $x$ TNM-4 y para P100S lo hicieron las cruzas TNM-1 x TNM-3, TNM-1 x TNM-5 y TNM-3 x TNM-4; del mismo modo, estas cruzas sobresalen con valores promedios altos en estas características (Tabla 7).

\section{CONCLUSIONES}

Los efectos de aptitud combinatoria general (efectos aditivos) fueron más importantes que los de aptitud combinatoria específica en la mayoría de los caracteres agronómicos; aunque en rendimiento de campo y aspecto general de planta destacaron los efectos de tipo no aditivo.

Se presentaron efectos recíprocos para $\mathrm{AP}$, DFF y AGP, lo que indica la importancia de seleccionar apropiadamente el progenitor femenino para favorecer el comportamiento agronómico en estos genotipos.

La línea TNM-5 presentó efectos altos de ACG en RC, AGP, APL, LM, DM y P100S, seguido por TNM-4 con estimadores altos y positivos para AGP, DM y P100S; además ambas líneas fueron más precoces que las demás, al mostrar estimadores negativos en DFM, DFF y DMF; por lo que estas líneas podrían ser mejoradas por algún método de selección recurrente para incrementar la frecuencia genética aditiva en características agronómicas y posteriormente usarse en 
programas de hibridación.

Finalmente, en las cruzas con estimadores altos y positivos en todas las características registradas participaron líneas con estimadores altos de ACG y con efectos bajos y negativos.

\section{LITERATURA CITADA}

Adel, M.M., and E.A. Ali. 2013. Gene action and combining ability in a six parent diallel cross of wheat. Asian J. Crop Sci. 5(1):14-23.

Aminu, D., S.G. Muhammed, and B.G. Kabir. 2014. Estimates of combining ability and heterosis for yield and yield traits in maize population (Zea mays L.) under drought conditions in the Northern Guinea and Sudan savanna zones of Borno State, Nigeria. Int. J. Agr. Res. Innovat. Tech. 2(5):824-830.

Aminu, D., and A.U. Izge. 2013. Gene action and heterosis for yield and yield traits in maize (Zea mays L.) under drought conditions in Northern guinea and Sudan savannas of Boron State, Nigeria. Peak Journal of Agricultural Science 1(1):17-23.

Andrio-Enríquez, E., F. Cervantes-Ortiz, J.G. Rivera-Reyes, L.P. Guevara-Acevedo, S.A. Rodríguez-Herrera, G. Rodríguez-Pérez, et al., 2015. Aptitud combinatoria y efectos maternos en razas de maíz Mexicanas por el método I de Griffing. Chil. J. Agric. Anim. Sci. 31(1):5-14.

Antuna Grijalba, O., S.A. Rodríguez Herrera, A. Espinoza Banda, P. Cano Rios, G. Llaven Valelncia, J.L. Coyac Rodríguez, et al., 2017. Componentes genéticos de caracteres agronómicos en razas de maíz y sus progenies híbridas. Phyton Int. J. Exp. Bot. 86:246-251.

Badu-Apraku, B., M. Oyekunle, R.O. Akinwale, and M. Aderounmu. 2013. Combining ability and genetic diversity of extra-early white maize inbreds under stress and non-stress environments. Crop Sci. 53:9-26.

Cervantes-Ortiz, F., G. García-de los Santos, A. Carballo-Carballo, D. Bergvinson, J. Crossa, M. Mendoza-Elos, et al. 2007. Herencia del vigor inicial de plántula y su correlación con caracteres de planta adulta en genotipos de maíz tropical. Agrociencia 41(4):425-433.

Cervantes-Ortiz, F., J. Hernández-Esparza, J.A. Rangel-Lucio, E. Andrio-Enríquez, M. Mendoza-Elos, G. Rodríguez-Pérez, et al. 2016. Aptitud combinatoria general y específica en la calidad de semilla de líneas $\mathrm{S}_{3}$ de maíz. Rev. Fit. Mex. 39(3):259-268.
Conrado, T.V., C.A. Scapim, L.S. Bignoto, R.J.B. Pinto, I.L.J. Freitas, A.T. Amaral, et al. 2014. Diallel analysis of corn for special use as corn grits: determining the main genetic effects for corn gritting ability. Genet. Mol. Res. 13:6548-6556.

De la Cruz-Lázaro, E., G. Castañón-Nájera, N.P. Brito-Manzano, A. Gómez-Vázquez, V. Robledo-Torres y A.J. Lozano-Del Río. 2010. Heterosis y aptitud combinatoria de poblaciones de maíz tropical. Phyton Int. J. Exp. Bot. 79:11-17.

Dhliwayo, T., K. Pixley, and V. Kazembe. 2005. Combining ability for resistance to maize weevil among 14 Southern African maize inbred lines. Crop. Sci. 45:662-667.

Dzib-Aguilar, A., J.C. Segura-Correa, R. Ortega-Paczka, y L. Latournerie-Moreno. 2011. Cruzas dialélicas entre poblaciones nativas de maíz de Yucatán y poblaciones mejoradas. Tropical and Subtropical Agroecosystems 14:119-127.

El-Badawy, M.E.M. 2013. Heterosis and combining ability in maize using diallel crossed among seven new inbred lines. Asian J. Crop Sci. 5:1-13.

Escorcia-Gutiérrez, N., J.D. Molina-Galán, F. Castillo-González, y J.A. Mejía-Contreras. 2010. Rendimiento, heterosis y depresión endogámica de cruzas simples de maíz. Rev. Fitotec. Mex. 33:271-279.

Esquivel G., F. Castillo, J.M. Hernández, A. Santacruz, G. García, y J.A. Acosta. 2013. Aptitud combinatoria en maíz con divergencia genética en el Altiplano Mexicano. Rev. Mex. Cienc. Agric. 4(1):5-18.

FAO. 2010. Invertir en seguridad alimentaria. 35 p. Food and Agriculture Organization (FAO), Viale delle Terme di Caracalla 00153, Roma, Italia.

Griffing, B. 1956. Concepts of general and specific combining ability in relation to diallel crossing systems. Aust. J. Biol. Sci. 9:463-493.

Guerrero, C., A. Espinoza, A. Palomo, E. Gutiérrez, H. Zermeño, y M.P. González. 2011. Aptitud combinatoria del rendimiento y sus componentes en dos grupos de líneas de maíz. Agron. Mesoam. 22(2):257-267.

Guillen-De la Cruz, P., E. De la Cruz-Lázaro, G. Castañón-Najera, R. Osorio-Osorio, N.P. Brito-Manzano, A. Lozano-del Rio, y U. López-Noverola. 2009. Aptitud combinatoria general y específica de germoplasma tropical de maíz. Tropical and Subtropical Agroecosystems 10:101-107.

Hansen, L. A., and J. R. Bagget. 1977. Reciprocal differences for plant and ear characteristics in sweet corn. Hort. Sci. 12:60-62. 
Haochuan, L., T. Jihua, H. Yanmin, Y. Jiwey, and L. Zonghua. 2014. Analysis on combining ability and estimation of genetic parameters for chlorophyll content in maize. J. Plant Breed. Crop Sci. 6(8):97-104.

Hernández-Esparza, J., J.A. Rangel-Lucio, E. Andrio-Enríquez, M. Mendoza-Elos, J.G. Rivera-Reyes, y F. Cervantes-Ortiz. 2015. Aptitud combinatoria para contenido de clorofila en líneas $S_{3}$ de maíz para el Bajío de México. Interciencia 40(11):789-793.

Hussain, M.A., and I.H. Ali. 2014. Combining ability, gene action and heterosis in some inbred lines of maize at two sowing dates using factorial mating design. International $\mathrm{J}$. Pure Appl. Sci. Tech. 21(1):17-30

Kalsy, H.S., and D. Sharma. 1972. Study of cytoplasmic effects in reciprocal crosses of divergent varieties of maize (Zea mays L.). Euphytica 21:527-533.

Khalid, M.D., A.H. Mohammad, F. Al-Falahy, and A.M. Abdel-Sattar. 2012. Genetic variations and gene effect controlling grain yield and some of its components in maize. J. Agr. Sci. Tech. 814-823.

Khehra, A.S., and S. K. Bhalla. 1976. Cytoplasmic effects on quantitative characters in maize (Zea mays L.). Theor. Appl. Genet. 47:271-274.

Luna, O.J., J.L.H. García, R.D.C. Valdés, M.A.R. Gallegos, P.R. Preciado, C.G. Guerrero, et al. 2013. Aptitud combinatoria y sus componentes genéticos en líneas de maíz. Universidad y Ciencia 29(3):243-253.

Mhike, X., D.M. Lungu, and B. Vivek. 2011. Combining ability studies amongst AREX and CIMMYT maize (Zea mays L.) inbred lines under stress and non stress conditions. Afr. J. Agr. Res. 6(8):1952-1957.

Mora, M., V. Ordaz, J.Z. Castellanos, A. Aguilar, F. Gavi, y V. Volke. 2001. Sistemas de labranza y sus efectos en algunas propiedades físicas en un vertisol, después de cuatro años de manejo. Terra 19:67-74.

Moterle, L.M., A.L. Braccini, C.A. Scapim, R.J.B. Pinto, L.S.A. Goncalves, A.T. do Amaral Jr., and T.R.C. Silva. 2013. Combining ability of tropical maize lines for seed quality and agronomic traits. Genet. Mol. Res. 10(3):22682278.

Ojo, G.O.S., D.K. Adedzwa, and L.L. Bello. 2007. Combining ability estimates and heterosis for grain yield and yield components in maize (Zea mays L.). J. Sustain. Develop. Agri. Env. 3:49-57.
Pérez-López, F.J., R. Lobato, J. García, J.D. Molina, J. López, y T. Cervantes. 2014. Líneas progenitoras de maíz de alto rendimiento como progenitores de híbridos de maíz de cruza simple. Agrociencia 48:425-437.

Pswarayi, A., and B.S. Vivek. 2008. Combining ability amongst CIMMYT's early maturing maize (Zea mays L.) germplasm under stress and non-stress conditions and identification of testers. Euphytica 162:353-362.

Silva, C.P.D., A. Teixeira Jr., A. Pio, H. Duarte, I. Laurenco de Jesus, C. Vittorazzi, et al. 2015. Combining ability between tropical and temperate popcorn lines for seed quality and agronomic traits. Aust. J. Crop Sci. 9(4):256-263.

Sprague, G.F., and A. Tatum. 1942. General vs. specific combining ability in single crosses of corn. J. Am. Soc. Agron. 34:923-32.

Sprague, G.F., and S. Eberhart. 1977. Corn and corn improvement. p. 305-363. American Society of Agronomy, Madison, Wisconsin, USA.

Tansey, G., y T. Rajotte. 2009. El Control Futuro de los Alimentos. 312 p. Mundi Prensa, Madrid, España.

Umar, U.U., S.G. Ado, D.A. Aba, and S.M. Bugaje. 2014. Combining ability, gene action and heterosis in maize (Zea mays L.) under drought stress. Int. J. Agric. Innov. Res. 3(3):953-958.

Vergara, A.N., S. Rodríguez, y H. Córdova. 2005. Aptitud combinatoria general y específica de líneas de maíz (Zea mays) tropical y subtropical. Agron. Mesoam. 16(2):137-143.

Wong, R.R., R.E. Gutiérrez, E.G.A. Palomo, S. Rodríguez, H. Córdoba, A.B. Espinoza, et al. 2007. Aptitud combinatoria de componentes del rendimiento en líneas de maíz para grano en la comarca lagunera, México. Rev. Fitotec. Mex. 30(2):181-189.

Zare, M., R. Choukan, E.M. Heravan, M.R. Bihamta, and K. Ordookhani. 2011. Gene action of some agronomic traits in corn (Zea mayz L.) using diallel cross analysis. Afr. J. Agric. Res. 6(3):693-703.

Zhang, Y., and M.S. Kang. 2003. Diallel-SAS: A program for Griffing's diallel methods. p. 1-19. In Kang, M.S. (ed.). Handbook of Formulas and Software for Plant Geneticists and Breeders. Food Product Press, New York, USA. 